\title{
Restoring global offset and lower limb length with a 3 offset option double- tapered stem
}

\author{
Stefano Biggi ${ }^{1,2^{*}}$ D, Lorenzo Banci ${ }^{3}$, Riccardo Tedino ${ }^{1,2}$, Andrea Capuzzo ${ }^{1,2}$, Gabriele Cattaneo ${ }^{1,2}$, \\ Stefano Tornago ${ }^{2}$ and Andrea Camera ${ }^{1,2}$
}

\begin{abstract}
Background: A proper restoration of hip biomechanics is fundamental to achieve satisfactory outcomes after total hip arthroplasty (THA). A global hip offset (GO) postoperatively reduction of more than $5 \mathrm{~mm}$ was known to impair hip functionality after THA. This study aimed to verify the restoration of the GO radiographic parameter after primary THA by the use of a cementless femoral stem available in three different offset options without length changing.

Methods: From a consecutive series of 201 patients (201 hips) underwent primary cementless THA in our center with a minimum 3-year follow up, 80 patients ( 80 hips) were available for complete radiographic evaluation for $\mathrm{GO}$ and limb length (LL) and clinical evaluation with Harris hip score (HHS). All patients received the same femoral stem with three different offset options (option A with $-5 \mathrm{~mm}$ offset, option B and option $C$ with $+5 \mathrm{~mm}$ offset, constant for each sizes) without changing stem length.

Results: Mean GO significantly increased by $+3 \mathrm{~mm}(P<0.05)$ and mean $\mathrm{LL}$ significantly decreased by $+5 \mathrm{~mm}$ $(P<0.05)$ after surgery, meaning that postoperatively the limb length of the operated side increased by +5 $\mathrm{mm}$. HHS significantly improved from 56.3 points preoperatively to 95.8 postoperatively $(P<0.001)$. Offset option A was used in 1 hip (1\%), B in 59 hips (74\%) and C in 20 hips (25\%).

Conclusions: The femur is lateralized with a mean of $+5 \mathrm{~mm}$ after surgery than, the native anatomy, whatever type of stem was used. Thus, the use of this 3-offset options femoral stem is effective in restoring the native biomechanical hip parameters as GO, even if 2 offset options were considered sufficient to restore GO.
\end{abstract}

Keywords: Total hip Arthroplasty, Offset, Global offset, Leg length, Hip parameters

\footnotetext{
* Correspondence: dott.sbiggi@gmail.com

${ }^{1}$ Clinica Città di Alessandria - Policlinico di Monza, via Moccagatta 30, 15122

Alessandria, AL, Italy

${ }^{2}$ Fondazione Lorenzo Spotorno - Onlus, Albenga, SV, Italy

Full list of author information is available at the end of the article
}

(C) The Author(s). 2020 Open Access This article is licensed under a Creative Commons Attribution 4.0 International License, which permits use, sharing, adaptation, distribution and reproduction in any medium or format, as long as you give appropriate credit to the original author(s) and the source, provide a link to the Creative Commons licence, and indicate if changes were made. The images or other third party material in this article are included in the article's Creative Commons licence, unless indicated otherwise in a credit line to the material. If material is not included in the article's Creative Commons licence and your intended use is not permitted by statutory regulation or exceeds the permitted use, you will need to obtain permission directly from the copyright holder. To view a copy of this licence, visit http://creativecommons.org/licenses/by/4.0/. The Creative Commons Public Domain Dedication waiver (http://creativecommons.org/publicdomain/zero/1.0/) applies to the data made available in this article, unless otherwise stated in a credit line to the data. 


\section{Background}

A proper restoration of joint biomechanical parameters is fundamental to achieve satisfactory outcomes in terms of hip functionality and patient's quality of life after total hip arthroplasty (THA).

The hip abductor muscles lever arm, which is mechanically the distance between the center of rotation of the hip and the insertion point of the muscles, is in fact strictly dependent by implant positioning which determines the reconstruction of the center of rotation, as well as the global hip offset or global femoral offset (GO) [1]. GO is the distance from the anatomical axis of the femur to the medial margin of the acetabulum and it is usually defined as the sum of the femoral offset (FO) and the acetabular offset (AO) [1]. FO is defined as the distance from the center of rotation of the femoral head to the anatomical axis of the femur [2]. AO is defined as the distance from the center of rotation of the femoral head to the perpendicular line passing through the medial edge of the ipsilateral teardrop [3].

When studying hip parameters restoration after primary THA, GO is more reliable as joint parameter than considering only FO because it takes into account also the acetabular cup placement.

It has been showed how a GO reduction of more than $5 \mathrm{~mm}$ is associated with less abductor muscle strength and decreased functional results after THA [4]. Femoral stems are usually available with different offset versions but not always this can be a suitable solution as these different options often change, not only the stem offset but also, at the same time, the stem length and, thus, limb length (LL). A traditional cementless doubletapered straight femoral stem available with three different offset options has been shown to be able to restore hip offset even if LL discrepancy is not always minimized postoperatively $[5,6]$.

This study aimed to verify the restoration of the ipsilateral $\mathrm{GO}$ and LL radiographic parameter after primary THA by the use of a cementless femoral stem available with three different offset options which do not change the stem length. Thus, the study hypothesis, that we want to reject, is that postoperative GO decreased by more than $5 \mathrm{~mm}$ in comparison to preoperative GO.

\section{Methods}

\section{Study cohort}

All consecutive patients who underwent primary THA using the same femoral stem from 2014 to 2015 at authors' previous institution (the Department of Prosthetic Surgery of the Santa Corona Hospital of Pietra Ligure - Italy) were identified through the hospital database.

If deemed suitable according to the inclusion / exclusion criteria of the study protocol, patients were contacted by telephone by one of the authors and invited to participate in the study by attending a follow-up visit.

Inclusion criteria were defined as diagnosis of unilateral primary or secondary osteoarthritis, avascular osteonecrosis of femoral head, mild dysplasia of the hip (Crowe I-III), cementless primary THA with the same femoral stem at the same center, same senior surgeon, 3-year minimum follow-up. Exclusion criteria were defined as history of hip surgery before THA, diagnosis of severe dysplasia of the hip (Crowe $\geq I V$ ) or femoral neck fracture, revision THA, cemented femoral stem, patients not able to walk, missing preoperative or postoperative radiographs.

Overall, 201 patients (201 hips), who met all inclusion/ exclusion criteria, were identified to be suitable to be enrolled in this study. Out of these 201 patients, 7 patients were dead for causes unrelated to the surgery and 40 patients were lost to follow-up, being unable to contact them by phone or mail. Thus, 154 patients were contacted to attend a follow-up visit: 80 patients were visited at the last follow-up with available preoperative and postoperative radiographs and the remaining 74 patients were called only by phone but without followup visit and postoperative radiographs.

At the follow-up visit, an objective evaluation of the operated hip was carried out with the Harris hip score (HHS) [7] completed on patient's current state of health, as well as with a radiographic evaluation and measurements for specific radiographic parameters. Any intraoperative and postoperative complication related to the implant and implant failure with components revision were recorded for femoral stem and implant survivals. All patients signed an informed consent form for their personal data recording and use. The study protocol has been approved by the competent ethics committee (protocol number AslAL.Orto.19.01).

\section{Prosthetic implant}

Before surgery, preoperative hip templating was always performed. All surgical procedures were performed by the senior author $(\mathrm{AC})$, using a posterolateral approach, without external rotators reconstruction and the patient placed in lateral position [8].

The same femoral component, the Synthesis femoral stem (Permedica S.p.A., Merate, Italy), was used in all cases. Synthesis is a conventional cementless sandblasted titanium femoral stem with a double-tapered straight wedge profile and a rectangular cross-section. Although sharing a similar design with the classic CLS Spotorno stem (Zimmer Inc. Warsaw, IN), the Synthesis stem has three different offset options for each of the 13 sizes (stem options A, B and C) without changing stem length, unlike the CLS Spotorno stem [9]. Compared to type B stem (standard option with $131^{\circ} \mathrm{CCD}$ angle and $0 \mathrm{~mm}$ offset lateralization), type A stem has a decreased offset by -5 
$\mathrm{mm}$, constant for each size, and type $\mathrm{C}$ stem has an increased offset by $+5 \mathrm{~mm}$, constant for each size (Fig. 1).

The assessment of the correct offset option was performed during preoperative templating and then confirmed

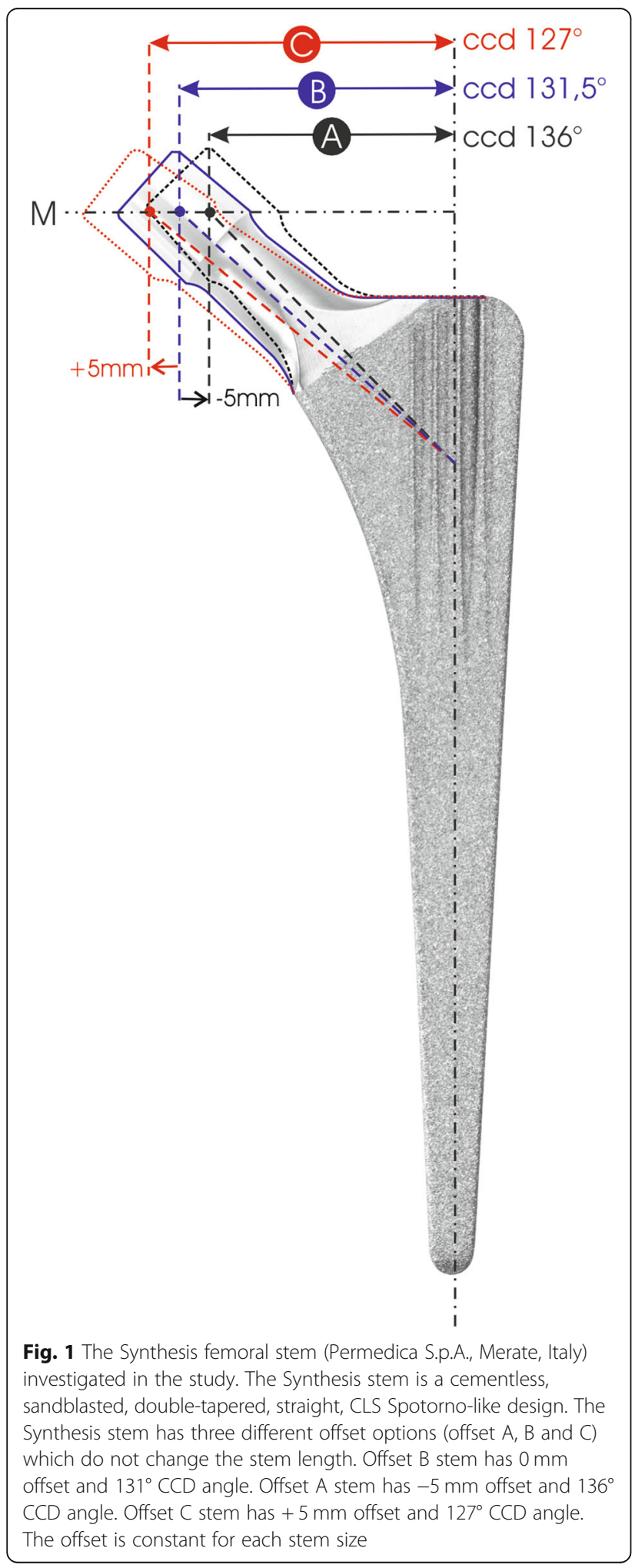

intraoperatively with trial components, considering hip morphotype and the possibility of correction of both limb length and hip offset.

Zirconia-reinforced alumina matrix (Biolox $x^{\circ}$ Delta CeramTec $\mathrm{GmbH}) 32 \mathrm{~mm}$-diameter femoral head coupled with a highly cross-linked ultra-high molecular weight polyethylene liner and a cementless porous tantalum acetabular shell (Zimmer Biomet) was used in most of the hips.

\section{Radiographic analysis}

Standard anteroposterior (AP) radiographs were taken preoperatively and postoperatively at the latest follow-up with the patient in supine position and both legs with $15^{\circ}$ of internal rotation. The X-ray beam was centered on the symphysis pubis. All radiographic measurements were taken twice, at different time points, on digital AP radiographs of the pelvis by the same author (SB), who was not involved in index surgery. Recorded values were the average of the two measurements.

Femur morphology was defined according to Dorr classification [10] and by measuring the Flare index [11] on the preoperative AP pelvis radiograph.

Femoral offset (FO), defined as the perpendicular distance from the center of rotation of the femoral head to the anatomical femoral axis [12], was measured preoperatively and postoperatively on AP pelvis radiographs. Acetabular offset (AO), defined as the perpendicular distance from the center of rotation of the femoral head to the line passing through the medial edge of the ipsilateral teardrop perpendicular to the line passing through the lower margins of the ischial tuberosity [13], was measured preoperatively and postoperatively on the AP pelvis radiographs. $\mathrm{GO}$ was measured as the sum of $\mathrm{FO}$ and $\mathrm{AO}$ [14]. GO difference $(\triangle G O)$ was defined as postoperative $G O$ - preoperative GO. Lower limb length (LL), defined as the distance between the medial apex of the ipsilateral lesser trochanter and the line passing through the lower margins of the ischial tuberosity [15], was measured preoperatively and postoperatively on AP pelvis radiographs. LL difference $(\Delta L L)$ was defined as postoperative $L L$ - preoperative LL.

Moreover, GO and LL were measured also on the contralateral hip to assess GO and LL discrepancies between the operated side and the contralateral side after surgery.

GO and LL distances were measured by using Horos Viewer software for MAC (Fig. 2).

Stem positioning, stem distal migration (subsidence), periprosthetic femoral fractures, periprosthetic endosteal bone formation (spotwelds), cortical hypertrophy, osteolysis, stress-shielding, pedestal formation under the tip of the stem and component stability were assessed on radiographs [16]. 


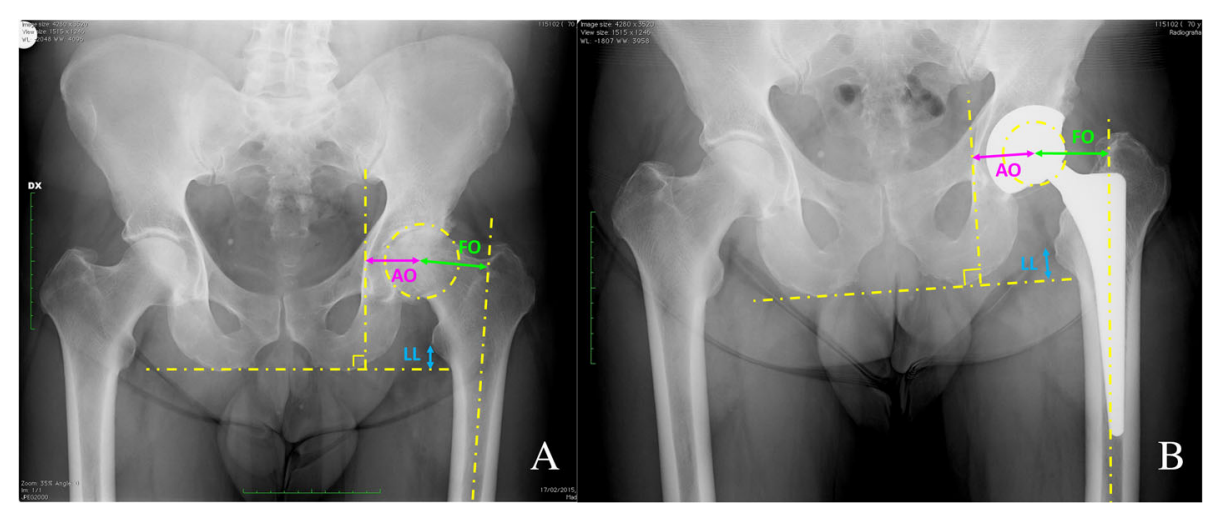

Fig. 2 Measurements of the hip biomechanical radiographic parameters by using Horos Viewer software for MAC at preoperative (a) and follow-up time (b). The global hip offset (GO) was defined by the sum of the femoral offset (FO) and the acetabular offset (AO). Limb length (LL) was defined as the distance between the medial apex of the ipsilateral lesser trochanter and the line passing through the lower margins of the ischial tuberosity

Stem subsidence was defined as femoral stem distal migration greater than $2 \mathrm{~mm}$ seen on the last AP radiograph in comparison to the immediately postoperative AP radiograph [17].

In particular, the radiolucency lines and osteolysis areas were reported and evaluated according to the Gruen method [18] on the femoral side.

\section{Other assessments}

Any complication occurred was recorded at the patient follow-up examination or at the telephone call.

The survival of the stem and the overall implant survival were analysed according to Kaplan Meier method [19], setting femoral component revision and any other components revision due to any reason and due to aseptic loosening as the end-point.

\section{Statistical analysis}

Descriptive statistical analysis was carried out to summarize the study results for each parameter considered. Continuous variables were reported by mean, maximum and minimum values and standard deviation. Dichotomous variables were reported by number and percentage. Analysis of continuous variables were performed with the t-Student test, whereas analysis of dichotomous variables with the chi-square test. Statistical significance was set with a $p<0.05$.

The study hypothesis to reject, was that $\Delta \mathrm{GO}$ resulted $<-5 \mathrm{~mm}$. Study alternative was that $\Delta \mathrm{GO}$ resulted $>5 \mathrm{~mm}$. Sample size analysis for 2 dependent groups (preoperatively vs postoperatively) using a difference of 5 $\mathrm{mm}$ for the primary endpoint variable $(\Delta \mathrm{GO})$ with a SD of $6 \mathrm{~mm}$ and alpha error of 0.05 required at least 15 subjects to include.

Statistical analysis was carried out with the MiniTab 17.2.1 statistical software.

\section{Results}

Mean (SD) age at surgery of the assessed patients was 72 (8) years. Mean (SD) BMI was 26.9 (3.9), 26 patients (32\%) were male and 54 patients (68\%) were female. Mean (SD) follow-up was 4.4 (0.6) years, ranging between 3.2 and 5.7 years. Diagnosis was primary osteoarthritis in 63 hips (82\%), avascular osteonecrosis in 8 hips (10\%), femoral neck fracture sequelae in 4 hips (5\%), mild hip dysplasia in 2 hips (1\%), others in 2 hips (1\%). Femur morphology was classified as type A in 26 hips (32\%), type $B$ in 51 hips (64\%), type $C$ in 3 hips (4\%), according to Dorr classification. Mean (SD) Flare index was 3.66 (0.70), ranging from 1.92 to 5.78 .

The type of Synthesis femoral stem used was A type in 1 hip (1\%), B type in 59 hips (74\%), $\mathrm{C}$ type in 20 hips (25\%).

Mean GO distance significantly increased by $+3 \mathrm{~mm}$ after surgery $(P<0.05) . \Delta G O$ was not $<-5 \mathrm{~mm}$, but within $\pm 5 \mathrm{~mm}$ range, then the alternative hypothesis was accepted and the null hypothesis was rejected (Table 1).

Mean LL distance significantly decreased by $-5 \mathrm{~mm}$ after surgery $(p<0.05)$, meaning that postoperatively the limb length of the operated side increased by $+5 \mathrm{~mm}$ (Table 1).

GO measured preoperatively in hips treated with type B stem was significantly lower $(p<0.043)$ in comparison with GO measured preoperatively in hips treated with type $C$ stem (Table 2). It means that the choice of the stem type used (type B or type $\mathrm{C}$ ) is actually dependent on preoperative hip offset. GO measured postoperatively did not differ $(p>0.05)$ between hips with type $\mathrm{B}$ and type $C$ stem (Table 2). Thus, the femur was lateralized after surgery at least as, or more than, the native anatomy, whatever type of stem was used.

LL measured postoperatively did not differ $(p>0.05)$ between hips with type $B$ stem and those with type $C$ 
Table 1 Analysis of the hip parameters compared between preoperative radiographs and postoperative radiographs. n.s. not statistically significant. n.a., not applicable

\begin{tabular}{|c|c|c|c|}
\hline Radiographic parameter & $\begin{array}{l}\text { Preoperative } \\
\text { Mean } \pm \text { SD (range) }\end{array}$ & $\begin{array}{l}\text { Postoperative } \\
\text { Mean } \pm \text { SD (range) }\end{array}$ & $p$-value \\
\hline GO (all hips) & $79.3 \pm 14.7(57-168) \mathrm{mm}$ & $81.8 \pm 19.1(58-230) \mathrm{mm}$ & 0.014 \\
\hline GO (offset B stem, 59 hips) & $77.8 \pm 15.8(57-168) \mathrm{mm}$ & $80.5 \pm 21.6(58-230) \mathrm{mm}$ & 0.048 \\
\hline GO (offset C stem, 20 hips) & $84.2 \pm 10.1(58-100) \mathrm{mm}$ & $86.3 \pm 8.6(74-110) \mathrm{mm}$ & n.s. \\
\hline GO (all contralateral hips) & $80.8 \pm 9.3(60-115) \mathrm{mm}$ & n.a. & \\
\hline LL (all hips) & $17.4 \pm 10.7(1-83) \mathrm{mm}$ & $11.9 \pm 9.8(1-60) \mathrm{mm}$ & 0.0001 \\
\hline LL (offset B stem, 59 hips) & $18.1 \pm 11.7(1-83) \mathrm{mm}$ & $11.5 \pm 8.6(1-46) \mathrm{mm}$ & 0.0001 \\
\hline LL (offset C stem, 20 hips) & $15.8 \pm 7.0(4-27) \mathrm{mm}$ & $13.9 \pm 12.6(1-60) \mathrm{mm}$ & n.s. \\
\hline LL (all contralateral hips) & $12.0 \pm 9.7(1-60) \mathrm{mm}$ & n.a. & \\
\hline
\end{tabular}

(Table 2). LL was not affected by the type of stem (which actually does not modify LL) but rather depends on the size of the stem used and the positioning and size of the cup.

HHS significantly improved from 56.3 points preoperatively to 95.8 postoperatively $(p<0.001)$.

There was no significant difference in postoperative HHS between patients with type $B$ stem and patients with type $C$ stem (respectively, 96.2 vs 95.2, $p>0.05$ ).

There were no significant differences for GO and LL between the operated side and the contralateral side after surgery, with a GO and a LL discrepancies of +1 $\mathrm{mm}$ and $-0.1 \mathrm{~mm}$, respectively (Table 1 ).

No stem subsidence was found at last follow-up. Presence of slight radiolucencies $(<2 \mathrm{~mm})$ due to proximal bone remodeling was observed mostly in zone 1 in 50 hips (62\%) and zone 7 in 11 hips (14\%). No femoral cortical hypertrophy was seen in any radiograph. Pedestal formations were present in 2 hips. Spotwelds were noted in $19(24 \%)$ hips predominantly in zone 3 and 5 . No periprosthetic osteolysis related to polyethylene debris was seen in any radiographs.

Two dislocations occurred postoperatively in 2 (0.9\%) patients. Both hips underwent revision surgery to replace the acetabular shell in one case and to replace the acetabular liner in the second case. In both revisions the femoral stem was left in situ. Other complications occurred were 1 (0.5\%) intertrochanteric femoral fracture occurred intraoperatively during stem placement and treated successfully with cerclage, 1 groin pain and 1 thigh pain. The number of complications did not differ between stem offset options (Table 3).

Survival of the whole implant was $98.9 \%$ at 6 -year follow-up with revision of any component for any reason as the endpoint. Survival of the femoral stem was $100 \%$ at 6-year follow-up with revision of the stem for any reason as the endpoint.

\section{Discussion}

The most important finding in this paper is that a double-tapered straight stem with three different offset options can restore the native anatomy and consequently the native biomechanical hip parameters, whatever type of stem is used. Moreover, at a short- to mid-term follow-up the survivorship of the stem is excellent.

Proper restoration of GO and hip center of rotation is crucial to achieve good and long-term results. The restoration of these parameters depends on a correct surgical technique. The hip abductor muscles lever arm is in fact strictly dependent by implant positioning which determines the reconstruction of the center of rotation, as well as GO. Al-Amiry et al. [1] showed that common errors in LL are mainly caused by improper femoral stem positioning, while

Table 2 Analysis of the radiographic parameters and the HHS compared between hips treated with offset B stem and hips treated with offset $C$ stem

\begin{tabular}{llll}
\hline Parameter & $\begin{array}{l}\text { Offset B stem (59 hips) } \\
\text { Mean (SD) }\end{array}$ & $\begin{array}{l}\text { Offset C stem (20 hips) } \\
\text { Mean (SD) }\end{array}$ & $\begin{array}{c}\boldsymbol{p} \text {-value } \\
\text { GO preop }\end{array}$ \\
GO postop & $77.8(16.0) \mathrm{mm}$ & $84.2(10.1) \mathrm{mm}$ & 0.043 \\
$\Delta$ GO & $80.5(21.8) \mathrm{mm}$ & $86.3(8.6) \mathrm{mm}$ & n.S. \\
LL preop & $2.7(10.0) \mathrm{mm}$ & $2.1(4.7) \mathrm{mm}$ & n.s. \\
LL postop & $18.1(11.7) \mathrm{mm}$ & $15.7(7.0) \mathrm{mm}$ & n.S. \\
$\Delta$ LL & $11.6(8.6) \mathrm{mm}$ & $13.9(12.6) \mathrm{mm}$ & n.s. \\
HHS postop & $-6.5(11.1) \mathrm{mm}$ & $-1.8(12.3) \mathrm{mm}$ & n.S. \\
\hline
\end{tabular}


Table 3 Details of the cases with complications

\begin{tabular}{lllllllll}
\hline Case & Age & Gender & FU & Complication & Revision & Stem Offset & $\Delta$ GO & GO discrepancy \\
\hline 1 & 76 & M & 4,8 & $\begin{array}{l}\text { Intraoperative intertrochanteric } \\
\text { fracture }\end{array}$ & No & C & $0 \mathrm{~mm}$ & $0 \mathrm{~mm}$ \\
2 & 69 & M & 4,2 & Dislocation & Yes, acetabular cup & C & $+10 \mathrm{~mm}$ & $-5 \mathrm{~mm}$ \\
3 & 64 & M & 4,7 & Dislocation & Yes, only acetabular liner & B & $+8 \mathrm{~mm}$ & $+3 \mathrm{~mm}$ \\
4 & 68 & M & 4,0 & Groin pain & No & C & $+2 \mathrm{~mm}$ & $+1 \mathrm{~mm}$ \\
5 & 75 & F & 3,5 & Thigh pain & No & B & $+10 \mathrm{~mm}+3 \mathrm{~mm}$ \\
\hline
\end{tabular}

global FO reduction results from improper positioning of both the femoral stem and the acetabular cup.

According to Bonnin et al. [20] GO should be restored to the native anatomy. Conventional acetabular preparation consists in medialization of the cup on the acetabular floor, leading to medialization of the hip center of rotation (HCR) and decreasing AO. If surgeon compensates the lack of $\mathrm{AO}$ by increasing $\mathrm{FO}$, this restores the $\mathrm{GO}$ and the abductor lever arm. Conversely, if surgeon only restores $\mathrm{FO}$, without regards to the $\mathrm{AO}, \mathrm{GO}$ and the abductor lever arm are reduced. Thus, the FO should be increased in order to compensate the lack of AO.

Some authors advocate the potential advantages in restoring the native $\mathrm{HCR}$ and therefore preserving $\mathrm{AO}$ [21-23]. Potential advantages include the improvement of acetabular bone stock, lower risk of bone or soft tissue impingement and dislocation, and improved hip kinematics. Similarly, disadvantages of preserving the acetabular offset include a potential decrease of the bone coverage of the acetabular component, a higher risk of anterior overhang (a source of iliopsoas tendon impingement), and an increase of the stress applied on the cup and on the femoral head $[22,23]$. Therefore, medialization of the cup gives biomechanical advantages according to Pawel's law and gives lower resulting forces at the headcup interface [20].

The restoration of $\mathrm{GO}$ should be performed in a range within $\leq \pm 5$ to $10 \mathrm{~mm}$ of native values, to gain better clinical scores, an adequate hip range of motion (ROM), gluteus strength and gait kinetics, and lower incidence of LL discrepancy [2, 4]. We agree with the authors, in fact before any surgery preoperative templating is always performed at our department, in order to choose the proper stem for each morphology of femur, limiting a LL discrepancy.

In our series GO and LL were restored within $\pm 5 \mathrm{~mm}$ of the native values, leading consequently to good clinical scores. No statistically significant differences in postoperative GO and LL were found in using type B or C stems, therefore the choice of different offset options seems to be related to hip morphology.

Innmann et al. [5] in their comparative study with three stem designs stated that the postoperative LLD was surgeon but not implant specific. Junior surgeons had slightly larger LL discrepancy, compared to senior surgeons $(2.4$ vs $1.2 \mathrm{~mm} ; P=0.043)$. However for both senior and junior surgeons, leg length reconstruction was most difficult with the CLS stem with a postoperative LL discrepancy of $2.6 \mathrm{~mm}$ (respectively, 1.6 vs 3.6 $\mathrm{mm}$ ) than with other types of stems, as a short curved stem (Fitmore, Zimmer-Biomet) with a postoperative LLD of $0.8 \mathrm{~mm}(P=0.002)$.

Also von Roth et al. [6] in their randomized controlled trial found at 2 weeks a significant difference in postoperative LLD between patients treated with a short curved stem (Fitmore, Zimmer-Biomet) and patients with the CLS stem (respectively, $-0.7 \mathrm{~mm}$ and $-2.9 \mathrm{~mm}, P=.01$ ).

The above-mentioned parameters are also related both with hip biomechanics and lower limb gait kinematics. Renkawitz et al. [24] confirmed, with their gait analysis of 60 patients with unilateral THA, that restoration of $\mathrm{LL}$ and GO within $\pm 5 \mathrm{~mm}$ increase ROM and gait kinematics. Conversely, the authors didn't found statistically significant distribution with the clinical scores and the restoration of LL and GO. Another gait analysis conducted by Stief et al. [25] showed that restoring GO correlate positively with the Knee Adduction Moment (KAM), so increasing offset, the knee has a greater varus moment during walking. This means that exceeding offset increase can both increase gluteus tension and hip stability, but, on the other hand, could worsen knee function. It is believed that lateral trochanteric pain may be the consequence of increased offset, but Abdulkarim et al. [26] found in their retrospective controlled case series that lateral trochanteric pain is not associated with implant positioning or increased offset.

Intraoperative femoral fractures with the Synthesis stem remain a risk during component implantation. We found 1 intraoperative intertrochanteric fracturing during stem placement. This specific complication related to this type of straight, double tapered, rectangular cross-sectional shaped design of femoral stem which appears to be reduced in our series in comparison with the classic CLS Spotorno design of which literature reported an incidence up to $4 \%[27,28]$ not depending by learning curve, stem offset or stem positioning [29].

This study, although confirming good clinical and radiographic results with $\mathrm{GO}$ restoring within $5 \mathrm{~mm}$ range, has 
some limits. First, it's retrospective with no control group. In some cases the affected hip in the preoperative radiographs were more externally rotated, due to the arthritic condition, thus the femoral neck seems more valgus and consequently the FO is decreased on the AP radiograph. In this study we compared preoperative parameters with postoperative reconstructed parameters. So, our control was the preoperative condition. It would be more interesting to have available a control group with a different design of stem, in order to directly compare the effectiveness in the restoration of GO and LL of different stem design. Finally, only two offset types of the same stem were used. Type A stem was used in only one case of hip dysplasia sequelae, with a short global offset, a mild deformity in neck anteversion and an increased CCD angle. In our series there were no valgus necks that could be matched with stem's conformation. Probably this offset option doesn't represent the morphology of some valgus necks, but our series is too restricted to assert it. Further prospective controlled studies with greater series are necessaries to achieve more reliable and reproducible results, but this paper shows encouraging outcomes.

\section{Conclusions}

The restoration of the biomechanical hip parameters after THA leads to satisfactory results. Surgical technique and preoperative planning are the first fundamental steps together with a stem with different offset options. The use of a traditional cementless double-tapered femoral stem, available with 3 offset options with no stem length changes, is effective in restoring the global femoral offset without any clinically significant change in lower limb length. Clinical results and implant survival are encouraging, despite the short follow-up.

\section{Abbreviations \\ THA: Total hip arthroplasty; GO: Global offset; FO: Femoral offset; AO: Acetabular offset; LL: Limb length; HHS: Harris hip score; AC: Andrea Camera; CLS: Cementless Lorenzo Spotorno; CCD: Centrum-collum- diaphyseal; AP: Antero-posterior; SB: Stefano Biggi; SD: Standard deviation; ROM: Range of motion; KAM: Knee adduction moment}

\section{Acknowledgements}

Not applicable.

\section{Authors' contributions}

AC (senior) performed all the surgical procedures; SB, AC, RT, GC and ST gave a major contribution in surgical time and at the follow-up; SB performed all the radiological measurements and gave contribution in writing the manuscript; LB gave major contribution in statistical analysis and writing the manuscript; all authors read and approved the final manuscript.

\section{Funding}

Not applicable.

\section{Availability of data and materials}

The data that support the findings of this study are available from "Fondazione Lorenzo Spotorno ONLUS" but restrictions apply to the availability of these data, which were used under license for the current study, and so are not publicly available. Data are however available from the authors upon reasonable request and with permission of "Fondazione Lorenzo Spotorno ONLUS".

\section{Ethics approval and consent to participate}

The study protocol has been approved by the "Comitato Etico Interaziendale" of the institute "ASO SS Antonio e Biagio e C. Arrigo", protocol number: AsIAL.Orto.19.01 del CE 21/03/2019. Informed Consent was obtained by submitting a written consent declaration form to the patient.

\section{Consent for publication}

Not applicable.

\section{Competing interests}

LB is employed as Clinical Affairs Manager by Permedica S.p.A., Via Como 38, 23807, Merate (LC), Italy. The other authors declare that they have no competing interests.

\section{Author details}

${ }^{1}$ Clinica Città di Alessandria - Policlinico di Monza, via Moccagatta 30, 15122 Alessandria, AL, Italy. ${ }^{2}$ Fondazione Lorenzo Spotorno - Onlus, Albenga, SV, Italy. ${ }^{3}$ Permedica S.p.A, Merate, LC, Italy.

Received: 19 June 2020 Accepted: 24 September 2020

Published online: 02 October 2020

\section{References}

1. Al-Amiry B, Mahmood S, Krupic F, Sayed-Noor A. Leg lengthening and femoral-offset reduction after total hip arthroplasty: where is the problem stem or cup positioning? Acta Radiol. 2017;58(9):1125-31.

2. Warnock J, Hill J, Humphreys L, Gallagher N, Napier R, Beverland D. Independent restoration of femoral and acetabular height reduces limb length discrepancy and improves reported outcome following total hip arthroplasty. J Orthop. 2019;16(6):483-8. https://doi.org/10.1016/j.jor.2019.05. 013 eCollection 2019 Nov-Dec.

3. Clement ND, S Patrick-Patel R, MacDonald D, Breusch SJ. Total hip replacement: increasing femoral offset improves functional outcome. Arch Orthop Trauma Surg. 2016;136(9):1317-23.

4. Mahmood SS, Mukka SS, Crnalic S, Wretenberg P, Sayed-Noor AS. Association between changes in global femoral offset after total hip arthroplasty and function, quality of life, and abductor muscle strength. A prospective cohort study of 222 patients. Acta Orthop. 2016;87(1):36-41.

5. Innmann MM, Spier K, Streit MR, Aldinger PR, Bruckner T, Gotterbarm T, Merle C. Comparative analysis of the reconstruction of individual hip anatomy using 3 different cementless stem designs in patients with primary hip osteoarthritis. J Arthroplast. 2018;33(4):1126-32.

6. von Roth P, Perka C, Mayr HO, Preininger B, Ziebula F, Matziolis G, Hube R. Reproducibility of femoral offset following short stem and straight stem total hip arthroplasty. Orthopedics. 2014;37(7):e678-84.

7. Harris WH. Traumatic arthritis of the hip after dislocation and acetabular fractures: treatment by mold arthroplasty. An end-result study using a new method of result evaluation. J Bone Joint Surg Am. 1969;51:737-55.

8. Gibson A. Posterior exposure of the hip joint. J Bone Joint Surg Br. 1950;32:183-6.

9. Aldinger PR, Breusch SJ, Lukoschek M, et al. a 10- to 15-year follow up of the cementless Spotorno stem. J Bone Joint surg Br. 2003;85:209-14.

10. Dorr LD, Faugere MC, Mackel AM, Gruen TA, Bognar B, Malluche HH. Structural and cellular assessment of bone quality of proximal femur. Bone. 1993;14(3):231-42.

11. Rubin PJ, Leyvraz PF, Aubaniac JM, Argenson JN, Estève P, de Roguin B. The morphology of the proximal femur. A three-dimensional radiographic analysis. J Bone Joint Surg Br. 1992;74(1):28-32.

12. Akiyama K, Nakata K, Kitada M, Yamamura M, Ohori T, Owaki H, Fuji T. Changes in axial alignment of the ipsilateral hip and knee after total hip arthroplasty. Bone Joint J. 2016;98-B(3):349-58.

13. Dastane M, Dorr LD, Tarwala R, Wan Z. Hip offset in total hip arthroplasty: quantitative measurement with navigation. Clin Orthop Relat Res. 2011; 469(2):429-36.

14. Flecher $\mathrm{X}$, Ollivier $\mathrm{M}$, Argenson JN. Lower limb length and offset in total hip arthroplasty. Orthop Traumatol Surg Res. 2016;102(1 Suppl):S9-20. 
15. Wylde V, Whitehouse SL, Taylor AH, Pattison GT, Bannister GC, Blom AW. Prevalence and functional impact of patient-perceived leg length discrepancy after hip replacement. Int Orthop. 2009;33:905-9.

16. Engh CA, Bobyn JD, Glassman AH. Porous-coated hip replacement. The factors governing bone ingrowth, stress shielding, and clinical results. J Bone Joint Surg Br. 1987;69(1):45e55.

17. Espehaug B, Havelin LI, Engesaeter LB, Vollset SE, Langeland N. Early revision among 12,179 hip prostheses. A comparison of 10 different brands reported to the Norwegian Arthroplasty register, 1987-1993. Acta Orthop Scand. 1995;66(6):487-93.

18. Gruen TA, Mcneice GM, Amstutz HC. "Modes of failure" of cemented stemtype femoral components: a radiographic analysis of loosening. Clin Orthop Relat Res. 1979;141:17-27.

19. Kaplan RL, Meier P. Nonparametric estimation from incomplete observations. J Am Stat Assoc. 1958:53:457.

20. Bonnin MP, Archbold PH, Basiglini L, Selmi TA, Beverland DE. Should the acetabular cup be medialised in total hip arthroplasty. Hip Int. 2011;21(4):428-35.

21. Beverland D. The transverse acetabular ligament: optimizing version. Orthopedics. 2010;33:631.

22. Archbold HA, Mockford B, Molloy D, McConway J, Ogonda L, Beverland D. The transverse acetabular ligament: an aid to orientation of the acetabular component during primary total hip replacement: a preliminary study of 1000 cases investigating postoperative stability. J Bone Joint Surg Br. 2006; 88:883-6.

23. Kurtz WB, Ecker TM, Reichmann WM, Murphy SB. Factors affecting bony impingement in hip arthroplasty. J Arthroplast. 2010;25:624-34.

24. Renkawitz T, Weber T, Dullien S, Woerner M, Dendorfer S, Grifka J, Weber M. Leg length and offset differences above $5 \mathrm{~mm}$ after total hip arthroplasty are associated with altered gait kinematics. Gait Posture. 2016;49:196-201.

25. Stief F, van Drongelen S, Brenneis M, Tarhan T, Fey B, Meurer A. Influence of hip geometry reconstruction on frontal plane hip and knee joint moments during walking following primary Total hip replacement. J Arthroplast. 2019; 34(12):3106-13. https://doi.org/10.1016/j.arth.2019.07.027 Epub 2019 Jul 27.

26. Abdulkarim A, Keegan C, Bajwa R, Sheehan E. Lateral trochanteric pain following total hip arthroplasty: radiographic assessment of altered biomechanics as a potential aetiology. Ir J Med Sci. 2018;187(3):663-8. https://doi.org/10.1007/s11845-017-1701-1 Epub 2018 Jan 15.

27. Streit MR, Schröder K, Körber M, Merle C, Gotterbarm T, Ewerbeck V, Aldinger PR. High survival in young patients using a second generation uncemented total hip replacement. Int Orthop. 2012;36(6):1129-36 Epub 2011 Nov 24.

28. Biemond JE, Pakvis DF, van Hellemondt GG, Buma P. Long-term survivorship analysis of the cementless Spotorno femoral component in patients less than 50 years of age. J Arthroplast. 2011;26(3):386-90 Epub 2010 Mar 29.

29. Timmer C, Gerhardt DMJM, de Visser E, de Kleuver M, van Susante JLC. High incidence of intraoperative calcar fractures with the cementless CLS Spotorno stem. Eur J Orthop Surg Traumatol. 2018;28(7):1291-6.

\section{Publisher's Note}

Springer Nature remains neutral with regard to jurisdictional claims in published maps and institutional affiliations.

Ready to submit your research? Choose BMC and benefit from:
- fast, convenient online submission
- thorough peer review by experienced researchers in your field
- rapid publication on acceptance
- support for research data, including large and complex data types
- gold Open Access which fosters wider collaboration and increased citations
- maximum visibility for your research: over 100M website views per year
At BMC, research is always in progress.
Learn more biomedcentral.com/submissions

